\title{
The Novel Bivariate Distribution: Statistical Properties and Real Data Applications
}

\author{
B. I. Mohammed, ${ }^{1,2}$ Abdulaziz S. Alghamdi $\left(\mathbb{D},{ }^{3}\right.$ Hassan M. Aljohani ${ }^{4},{ }^{4}$ \\ and Md. Moyazzem Hossain $\mathbb{D i D}^{5}$ \\ ${ }^{1}$ Department of Mathematics, Faculty of Science, Jeddah University, 2749 Asfan Rd., Jeddah 21589, Saudi Arabia \\ ${ }^{2}$ Department of Mathematics, Faculty of Science, Al- Azhar University, Nasr City, Cairo, Egypt \\ ${ }^{3}$ Department of Mathematics, College of Science \& Arts, King Abdulaziz University, P. O. Box 344, Rabigh 21911, Saudi Arabia \\ ${ }^{4}$ Department of Mathematics \& Statistics, College of Science, Taif University, P.O. Box 11099, Taif 21944, Saudi Arabia \\ ${ }^{5}$ Department of Statistics, Jahangirnagar University, Savar, Dhaka-1342, Bangladesh
}

Correspondence should be addressed to Md. Moyazzem Hossain; hossainmm@juniv.edu

Received 27 October 2021; Revised 17 November 2021; Accepted 22 November 2021; Published 15 December 2021

Academic Editor: Saima K Khosa

Copyright (C) 2021 B. I. Mohammed et al. This is an open access article distributed under the Creative Commons Attribution License, which permits unrestricted use, distribution, and reproduction in any medium, provided the original work is properly cited.

\begin{abstract}
This article proposes a novel class of bivariate distributions that are completely defined by stating their conditionals as Poisson exponential distributions. Numerous statistical properties of this distribution are also examined here, including the conditional probability mass function (PMF) and moments of the new class. The techniques of maximum likelihood and pseudolikelihood are used to estimate the model parameters. Additionally, the effectiveness of the bivariate Poisson exponential conditional (BPEC) distribution is compared to that of the bivariate Poisson conditional (BPC), the bivariate Poisson (BP), the bivariate Poisson-Lindley (BPL), and the bivariate negative binomial (BNB) distributions using a real-world dataset. The findings of Akaike information criterion (AIC) and Bayesian information criterion (BIC) reveal that the BPEC distribution performs better than the other distributions considered in this study. As a result, the authors claim that this distribution may be used to fit dependent and overspread count data.
\end{abstract}

\section{Introduction}

In many areas of application, it is appropriate to study discrete bivariate variables. For example, problems arise in many social, economic, and physical phenomena [1], and in insurance risk applications, those number of cases in distinctive classifications will be regularly randomized (the readers are referred to $\mathrm{Wu}$ and Yuen [2], Yuen et al. [3], and Morata [4] for more details). Several authors have discussed these problems from different points of view, which include traffic accidents by Cacoullos and Papageorgiou [5] and Papageorgiou $[6,7]$ and the problem associated with crime utilizing the method of Miethe et al. [8]. Also, Lee [9] and Karlis and Ntzoufras [10] modeled scores "points and goals" of two competing teams in sports and pointed out that they are highly correlated. Modeling dependence on goals scored by teams competing in international football matches was studied by McHale and Scarf [11], and evaluated risks and spot errors using scarce data were discussed by Ahooyi et al. [12]. Several discrete bivariate models have been proposed in the literature (see, for example, Marshall and Olkin [13], Mishra [14], Özel [15-17], Reilly and Sapkota [18], Lee and Cha [19], and Jiang et al. [20]). The specific conditional distributions are one of the most important ways to get flexible bivariate distributions. Moreover, the important role of functional equations has been emphasized in establishing results in this regard which is highlighted by Castillo and Galambos [21-23], Arnold [24], Arnold et al. [25-27], Kottas et al. [28], and Gharib and Mohammed [29]. The use of this type of distribution in risk analysis and economics is relatively new; however, some applications were done by Sarabia et al. [30, 31].

In this paper, another class of bivariate model for Poisson exponential conditionals will be considered. A 
discrete random variable $X$ is said to have a one-parameter Poisson exponential distribution (PED) for modeling countable data if its probability mass function (PMF) is

$$
P(Z=z)=\frac{\alpha}{(1+\alpha)^{z}}, \quad \alpha>0, z=0,1, \ldots
$$

If the parameter of the Poisson model follows a continuous exponential distribution, then equation (1) is a mixture of Poisson and exponential distributions denoted by $Z \sim \operatorname{PE}(\alpha)$. This distribution is applicable to biological datasets, traffic datasets, thunderstorm datasets, and other discrete datasets. The scientific properties and estimation for parameter have been examined by Fazal and Bashir [32]; also, its requisition turns out that it will be a great substitution cost from claiming Poisson and Lindley distributions. In this paper, a new class of bivariate distribution has been proposed which is fully characterized by specifying its conditionals as Poisson exponential distribution. Finally, the performance of this distribution is compared with other distributions considering a real-life dataset.

\section{Bivariate Poisson Exponential Conditionals}

Consider a general bivariate model $(J, K)$ whose conditional distributions must satisfy the following two conditions:

$$
\begin{gathered}
J \mid K=k \sim \operatorname{PE}\left(\eta_{1}(k)\right), \\
K \mid J=j \sim \operatorname{PE}\left(\eta_{2}(j)\right),
\end{gathered}
$$

where $\eta_{1}(k)$ and $\eta_{2}(j)$ are some positive functions and PE denotes a Poisson exponential distribution. These equations lead us to discuss the next theorem.

Theorem 1. The discrete bivariate model with $J \mid K=k \sim P E\left(\eta_{1}(k)\right)$ and $K \mid J=j \sim P E\left(\eta_{2}(j)\right)$ can be described by the following distribution:

$$
\begin{aligned}
P_{J, K}(j, k) & =\left[N\left(\theta_{1}, \theta_{2}, \theta_{3}\right)\right]^{-1} \exp \left[\theta_{1} k-\theta_{3} j k+\theta_{2} j\right], \\
j, k & =0,1, \ldots, \theta_{1}, \theta_{2}<0, \theta_{3} \in \mathscr{R},
\end{aligned}
$$

where $\left[N\left(\theta_{1}, \theta_{2}, \theta_{3}\right)\right]^{-1}$ is the normalizing constant such that $P_{J, K}(j, k)$ summates to 1 .

Proof. According to (2) and (3), we can write the joint density $P(j, k)$ as a product of a marginal and a conditional density in both ways to get

$$
\left(\frac{\eta_{1}(k)}{\left(1+\eta_{1}(k)\right)^{j}}\right) h_{K}(k)=\left(\frac{\eta_{2}(j)}{\left(1+\eta_{2}(j)\right)^{k}}\right) h_{J}(j),
$$

where $h_{J}(j)$ and $h_{K}(k)$ are the marginal PMFs of $J$ and $K$, respectively.

Denoting

$$
\begin{gathered}
g(k)=\log \left[\eta_{1}(k) h_{K}(k)\right], \\
f(j)=\log \left[\eta_{2}(j) h_{J}(j)\right],
\end{gathered}
$$

equation (5) readily reduces to

$$
g(k)-x \log \left(1+\eta_{1}(k)\right)-f(j)-k \log \left(1+\eta_{2}(j)\right)=0,
$$

which is a special case of the functional equation $\sum_{m=1}^{n} f_{m}(x) g_{m}(y)=0$, whose most general solution is given by Aczel [33] as follows:

$$
\alpha_{2}(j)=\exp \left(-\theta_{1}+\theta_{3} j\right)-1, \ldots, \eta_{1}(k)=\exp \left(-\theta_{2}+\theta_{3} k\right)-1 \text {. }
$$

Substituting these expressions in (6) and (7), we can get the marginal PMF as

$$
\begin{gathered}
h_{J}(j)=\frac{\left[N\left(\theta_{1}, \theta_{2}, \theta_{3}\right)\right]^{-1}}{\exp \left(-\theta_{1}+\theta_{3} j\right)-1} \exp \left(-\theta_{2} j\right), \quad j=0,1, \ldots, \\
h_{K}(k)=\frac{\left[N\left(\theta_{1}, \theta_{2}, \theta_{3}\right)\right]^{-1}}{\exp \left(-\theta_{2}+\theta_{3} k\right)-1} \exp \left(-\theta_{1} k\right), \quad k=0,1, \ldots
\end{gathered}
$$

Finally, in accordance with (10) and (11), the class of discrete bivariate distribution with Poisson exponential conditionals is that given by (4), which describes the complete class of the BPEC distribution that has the three parameters $\theta_{1}, \theta_{2}$ (intensity parameters for $K$ and $J$, respectively), and $\theta_{3} \in \mathscr{R}$ (interaction or dependence parameter), where $\theta_{3}=0$ corresponds to independence between $J$ and $K$.

Figure 1 shows the three-dimensional curve of the BPLC given by (4) for the special cases for $\theta_{1}, \theta_{2}$, and $\theta_{3}$.

\section{Properties of the Bivariate Poisson Exponential Class}

In this part, the fundamental properties of the new bivariate distribution are contemplated.

We first know that the class (4) has the three parameters $\theta_{1}, \theta_{2}$, and $\theta_{3}$, while $\left[N\left(\theta_{1}, \theta_{2}, \theta_{3}\right)\right]^{-1}$ is the normalizing constant and is given by

$$
\begin{aligned}
N\left(\theta_{1}, \theta_{2}, \theta_{3}\right) & =\sum_{j=0}^{\infty} \sum_{k=0}^{\infty} \operatorname{Exp}\left[\theta_{1} k-\theta_{3} j k+\theta_{2} j\right] \\
& =\sum_{j=0}^{\infty} \frac{e^{-\theta_{1}+\left(\theta_{3}-\theta_{2}\right) j}}{e^{\theta_{1}+\theta_{3} j}-1} .
\end{aligned}
$$

3.1. Conditional PMF and Moments. The particular manifestations of the conditional distributions to the new model would provide

$$
\begin{aligned}
& P_{J \mid K}(j \mid k)=\frac{\exp \left(-\theta_{2}+\theta_{3} k\right)-1}{\left(\exp \left(-\theta_{2}+\theta_{3} k\right)\right)^{j}}, \quad j, k=0,1, \ldots, \\
& P_{K \mid J}(k \mid j)=\frac{\exp \left(-\theta_{1}+\theta_{3} j\right)-1}{\left(\exp \left(-\theta_{1}+\theta_{3} j\right)\right)^{k}}, \quad j, k=0,1, \ldots,
\end{aligned}
$$




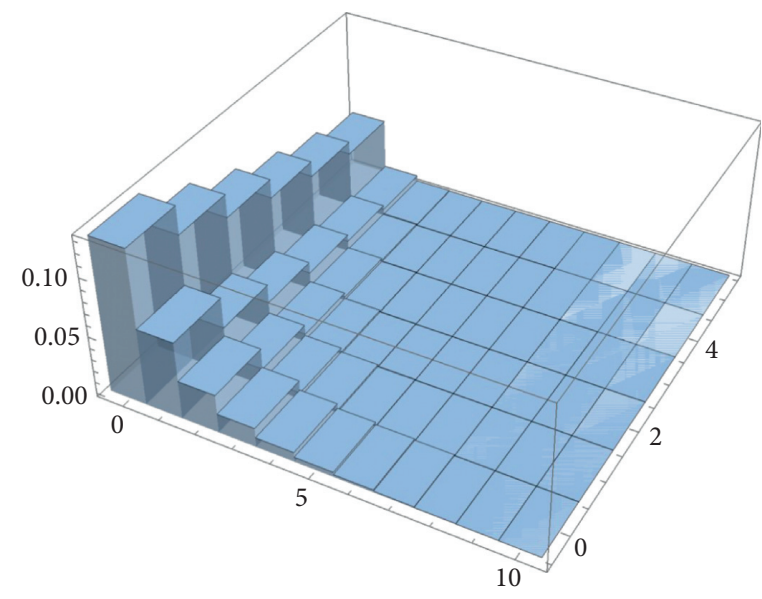

(a)

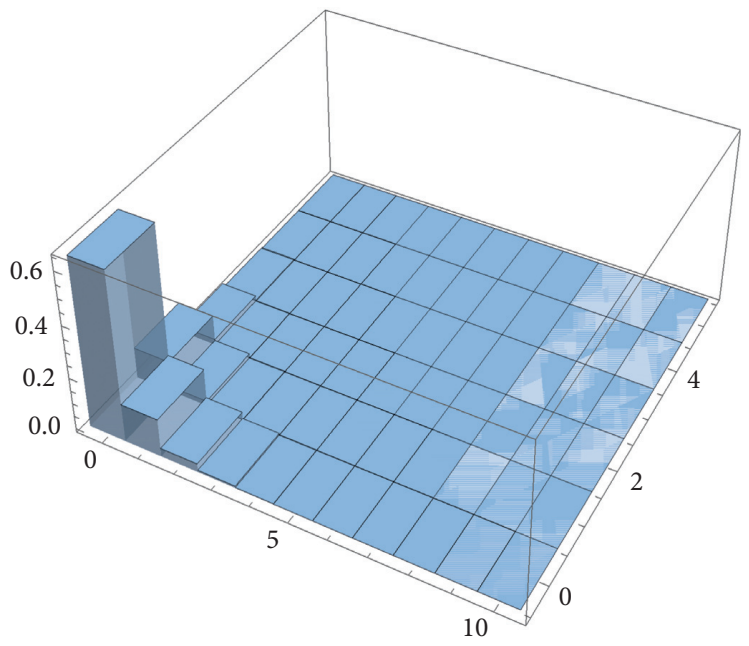

(c)

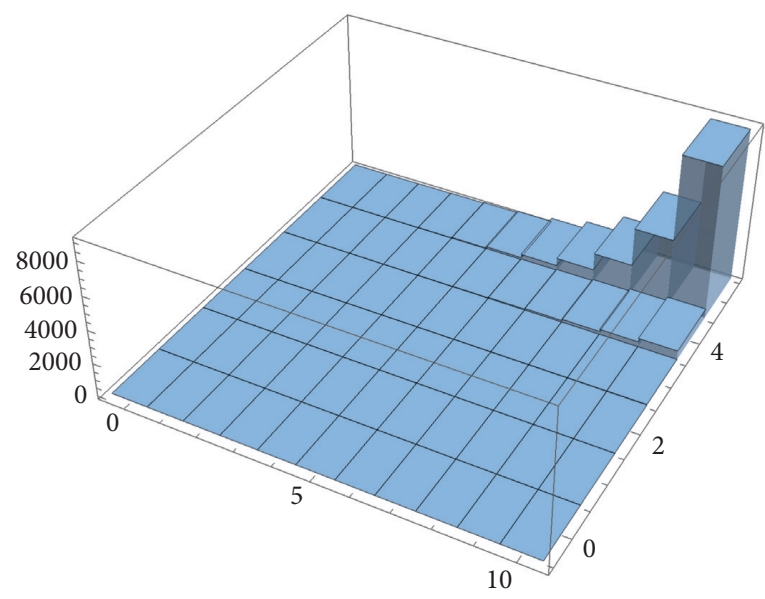

(b)

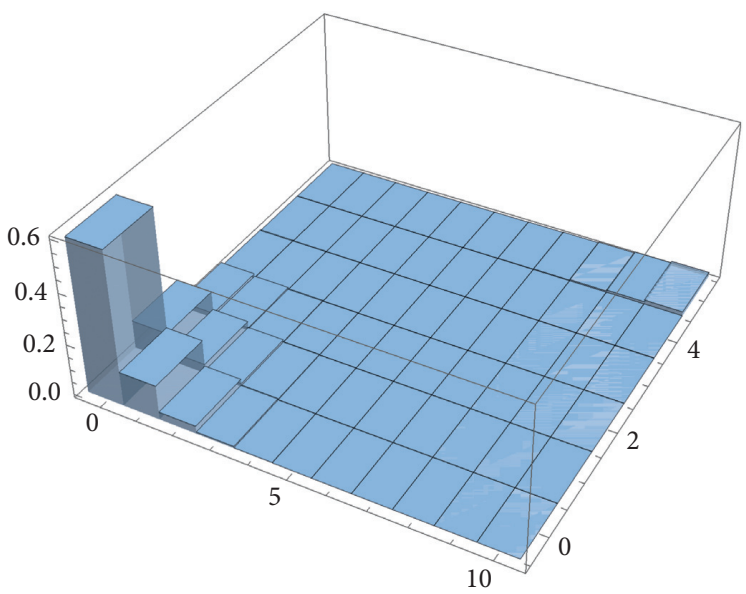

(d)

FIgURE 1: The three-dimensional curve of the BPLC under different scenarios. (a) BPEC with $\theta_{1}=-0.2, \theta_{2}=-0.7$, and $\theta_{3}=0.3$. (b) BPEC with $\theta_{1}=-0.2, \theta_{2}=-0.7$, and $\theta_{3}=-0.3$. (c) BPEC with $\theta_{1}=-1.7, \theta_{2}=-1.5$, and $\theta_{3}=0.4$. (d) BPEC with $\theta_{1}=-1.7, \theta_{2}=-$ 1.5 , and $\theta_{3}=-0.4$.

i.e.,

$$
\begin{aligned}
& J \mid K=k \sim P E\left(\exp \left(-\theta_{2}+\theta_{3} k\right)-1\right), \\
& K \mid J=j \sim \operatorname{PE}\left(\exp \left(-\theta_{1}+\theta_{3} j\right)-1\right) .
\end{aligned}
$$

The conditional distributions are given by (13) and (14), satisfying the compatibility conditions, and are studied by Arnold et al. [26], which guarantees the existence of the discrete bivariate model (4).

The regression functions for these conditional distributions are

$$
\begin{aligned}
& E(J \mid K=k)=\frac{1}{\exp \left(-\theta_{2}+\theta_{3} k\right)-1}, \\
& E(K \mid J=j)=\frac{1}{\exp \left(-\theta_{1}+\theta_{3} j\right)-1} .
\end{aligned}
$$

These regression functions are nonlinear and decreasing (increasing) if $\theta_{3}>0\left(\theta_{3}<0\right)$ (see Figure 2).
The first moment of the pair $(J, K)$ is obtained by direct calculations using (4), and we find that

$$
E(J K)=\sum_{j=0}^{\infty} \frac{\left[N\left(\theta_{1}, \theta_{2}, \theta_{3}\right)\right]^{-1} j e^{-\left(\theta_{1}+\left(\theta_{2}+\theta_{3}\right) j\right)}}{\left(e^{-\theta_{1}}+e^{\theta_{3} j}\right)^{2}} .
$$

Special Classes. Class (4) can be classified by suitable selections for the parameters $\theta_{1}, \theta_{2}$, and $\theta_{3}$ into the following two subclasses.

(a) Subclass I (subclass with two parameters):

$$
\begin{array}{r}
P_{J, K}(j, k)=\left[N\left(\theta_{1}, \theta_{2}, \theta_{3}\right)\right]^{-1} \exp \left[\theta_{1} k-\theta_{3} j k+\theta_{2} j\right], \\
j, k=0,1, \ldots, \theta_{1}, \theta_{2}<0, \theta_{3} \in \mathscr{R} .
\end{array}
$$

(1) $\theta_{3}=0, J, K$ are independent, and (4) reduces to 

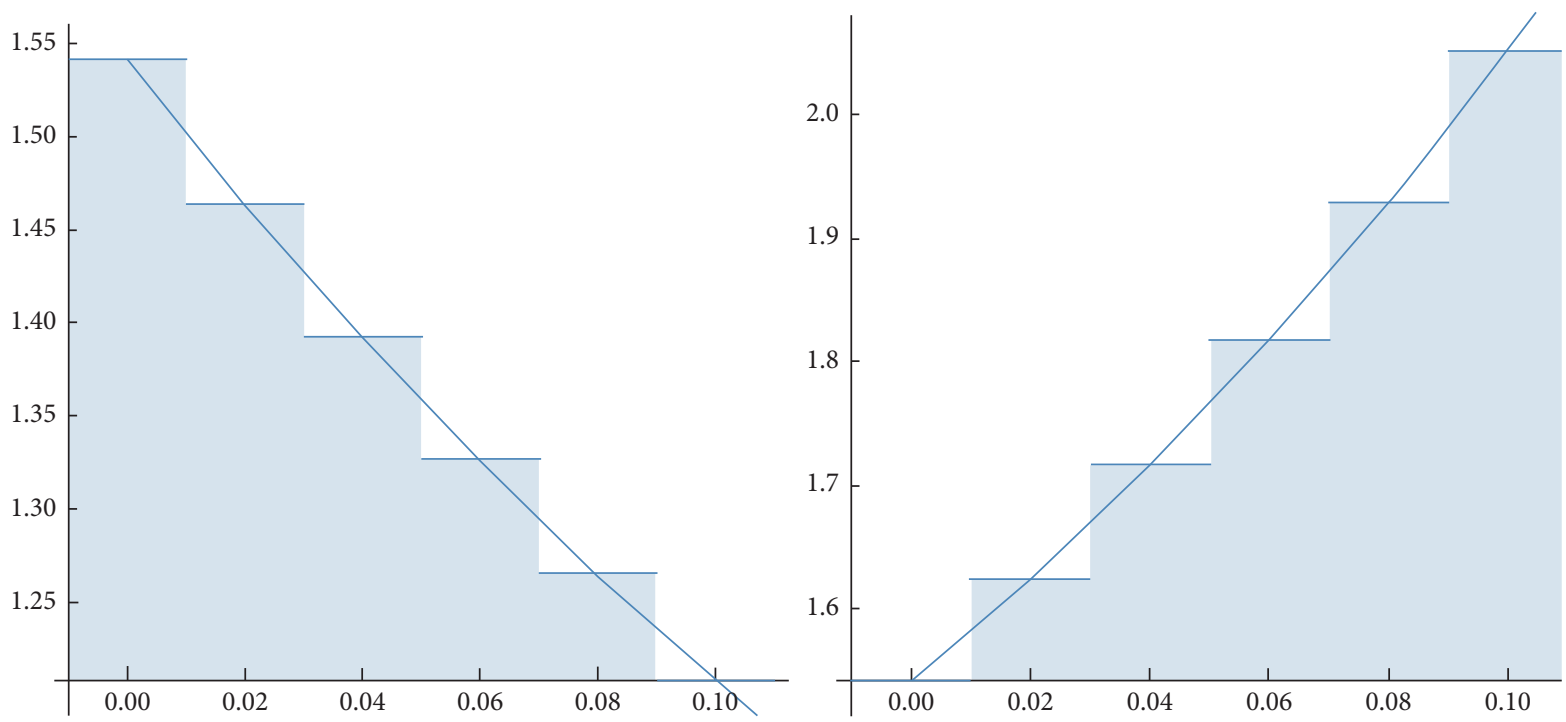

FIgure 2: The regression curve of $j$ on $k(k$ on $j)$ of BPEC distribution for $\theta_{3}=0.5>0\left(\theta_{3}=-0.5<0\right)$.

$$
\begin{aligned}
P_{J, K}(j, k) & =\left[N\left(\theta_{1}, \theta_{2}, 0\right)\right]^{-1} \exp \left(\theta_{2} j\right) \exp \left(\theta_{1} k\right) ; \\
j, k & =0,1, \ldots, \theta_{1}<0, \theta_{2} \in \mathscr{R},
\end{aligned}
$$

where $\left[N\left(\theta_{1}, \theta_{2}, 0\right)\right]^{-1}=1 /\left(e^{\theta_{1}}-1\right)\left(e^{\theta_{2}}-1\right)$.

It is clear from (19) that the two random variables (RVs) $J$ and $K$ are independent, with the following marginal densities:

$$
\begin{aligned}
& P_{J \mid K}(j \mid k)=\frac{\exp \left(-\theta_{2}\right)-1}{\left(\exp \left(-\theta_{2}\right)\right)^{j}}, \quad j, k=0,1, \ldots, \\
& P_{K \mid J}(k \mid j)=\frac{\exp \left(-\theta_{1}\right)-1}{\left(\exp \left(-\theta_{1}\right)\right)^{k}}, \quad j, k=0,1, \ldots,
\end{aligned}
$$

i.e.,

$$
\begin{aligned}
& J \mid K=k \sim \operatorname{PE}\left(\exp \left(-\theta_{2}\right)-1\right), \\
& K \mid J=j \sim \operatorname{PE}\left(\exp \left(-\theta_{1}\right)-1\right) .
\end{aligned}
$$

(2) $\theta_{1}=\theta_{2}$, and (4) reduces to

$$
\begin{aligned}
P_{J, K}(j, k) & =\left[N\left(\theta_{2}, \theta_{2}, \theta_{3}\right)\right]^{-1} \exp \left[(j+k) \theta_{2}-\theta_{3} j k\right], \\
j, k & =0,1, \ldots, \theta_{2}<0, \theta_{3} \in \mathscr{R},
\end{aligned}
$$

where

$$
\begin{aligned}
{\left[N\left(\theta_{2}, \theta_{2}, \theta_{3}\right)\right]^{-1} } & =\sum_{x=0}^{\infty} \frac{e^{\left(\theta_{2}+\theta_{3}\right) j}}{-e^{\theta_{2}}+e^{\theta_{3} j}}, \\
P_{J \mid K}(j \mid k) & =\frac{\exp \left(-\theta_{2}+\theta_{3} k\right)-1}{\left(\exp \left(-\theta_{2}+\theta_{3} k\right)\right)^{j}}, \quad j, k=0,1, \ldots, \\
P_{K \mid J}(k \mid j) & =\frac{\exp \left(-\theta_{1}+\theta_{3} j\right)-1}{\left(\exp \left(-\theta_{1}+\theta_{3} j\right)\right)^{k}}, \quad j, k=0,1, \ldots,
\end{aligned}
$$

i.e.,

$$
\begin{aligned}
& J \mid K=k \sim \operatorname{PE}\left(\exp \left(-\theta_{2}+\theta_{3} k\right)-1\right), \\
& K \mid J=j \sim \operatorname{PE}\left(\exp \left(-\theta_{2}+\theta_{3} j\right)-1\right) .
\end{aligned}
$$

(3) $\theta_{2}=0$, and (4) becomes

$$
\begin{aligned}
P_{J, K}(j, k) & =\left[N\left(\theta_{1}, 0, \theta_{3}\right)\right]^{-1} \exp \left[\theta_{1} k-\theta_{3} j k\right], \\
j, k & =0,1, \ldots, \theta_{1}<0, \theta_{3} \in \mathscr{R},
\end{aligned}
$$

where

$$
\left[N\left(\theta_{1}, 0, \theta_{3}\right)\right]^{-1}=\sum_{x=0}^{\infty} \frac{e^{\theta_{3} j}}{-e^{\theta_{1}}+e^{\theta_{3} j}}
$$

$$
\begin{aligned}
& P_{J \mid K}(j \mid k)=\frac{\exp \left(\theta_{3} k\right)-1}{\left(\exp \left(\theta_{3} k\right)\right)^{j}}, \quad j, k=0,1, \ldots, \\
& P_{K \mid J}(k \mid j)=\frac{\exp \left(-\theta_{1}+\theta_{3} j\right)-1}{\left(\exp \left(-\theta_{1}+\theta_{3} j\right)\right)^{k}}, \quad j, k=0,1, \ldots,
\end{aligned}
$$

i.e.,

$$
\begin{aligned}
& J \mid K=k \sim \operatorname{PE}\left(\exp \left(\theta_{3} k\right)-1\right), \\
& K \mid J=j \sim \operatorname{PE}\left(\exp \left(-\theta_{1}+\theta_{3} j\right)-1\right) .
\end{aligned}
$$

(4) $\theta_{1}=0$, and (4) reduces to

$$
\begin{aligned}
P_{J, K}(j, k) & =\left[N\left(0, \theta_{2}, \theta_{3}\right)\right]^{-1} \exp \left[-\theta_{3} j k+\theta_{2} j\right], \\
j, k & =0,1, \ldots, \theta_{2}<0, \theta_{3} \in \mathscr{R},
\end{aligned}
$$

where 


$$
\begin{aligned}
{\left[N\left(0, \theta_{2}, \theta_{3}\right)\right]^{-1} } & =\sum_{k=0}^{\infty} \frac{e^{\theta_{3} k}}{-e^{\theta_{2}}+e^{\theta_{3} k}}, \\
P_{J \mid K}(j \mid k) & =\frac{\exp \left(-\theta_{2}+\theta_{3} k\right)-1}{\left(\exp \left(-\theta_{2}+\theta_{3} k\right)\right)^{j}}, \quad j, k=0,1, \ldots, \\
P_{K \mid J}(k \mid j) & =\frac{\exp \left(\theta_{3} j\right)-1}{\left(\exp \left(\theta_{3} j\right)\right)^{k}}, \quad j, k=0,1, \ldots,
\end{aligned}
$$

i.e.,

$$
\begin{aligned}
& J \mid K=k \sim \operatorname{PE}\left(\exp \left(-\theta_{2}+\theta_{3} k\right)-1\right), \\
& K \mid J=j \sim \operatorname{PE}\left(\exp \left(\theta_{3} j\right)-1\right) .
\end{aligned}
$$

(b) Subclass II (subclass with one parameter):

(1) $\theta_{1}=\theta_{2}=\theta_{3}, \theta_{3}>0$, and (4) reduces to

$$
\begin{aligned}
P_{J, K}(j, k) & =\left[N\left(\theta_{3}\right)\right]^{-1} \exp \left[(j-j k+k) \theta_{3}\right], \\
j, k & =0,1, \ldots, \theta_{3}<0,
\end{aligned}
$$

where

$$
\begin{aligned}
{\left[N\left(\theta_{3}\right)\right]^{-1} } & =\sum_{y=0}^{\infty} \frac{e^{2 \theta_{3} k}}{-e^{\theta_{3}}+e^{\theta_{3} k}}, \\
P_{J \mid K}(j \mid k) & =\frac{\exp \left[(k-1) \theta_{3}\right]-1}{\left(\exp \left[(k-1) \theta_{3}\right]\right)^{j}}, \quad j, k=0,1, \ldots, \\
P_{K \mid J}(k \mid j) & =\frac{\exp \left[(j-1) \theta_{3}\right]-1}{\left(\exp \left[(j-1) \theta_{3}\right]\right)^{k}}, \quad j, k=0,1, \ldots, \\
\text { i.e., } \quad & \\
J \mid K & =k \sim \operatorname{PE}\left(\exp \left[(k-1) \theta_{3}\right]-1\right), \\
K \mid J & =j \sim \operatorname{PE}\left(\exp \left((j-1) \theta_{3}\right)-1\right) .
\end{aligned}
$$

\section{Estimation of the Parameters of BPLC}

Suppose that $\left(j_{1}, k_{1}\right),\left(j_{2}, k_{2}\right), \ldots,\left(j_{n}, k_{n}\right)$ are random samples from $\operatorname{BPEC}\left(\theta_{1}, \theta_{2}, \theta_{3}\right)$ class with density function given in (4).

4.1. Maximum Likelihood Estimation (MLE) for the Parameters. The log-likelihood function $l(\theta)$ of $\operatorname{BPEC}\left(\theta_{1}, \theta_{2}, \theta_{3}\right)$ is given by

$$
l(\theta)=-n \log \left(N\left(\theta_{1}, \theta_{2}, \theta_{3}\right)\right)+\theta_{1} \sum_{i=1}^{n} k_{i}-\theta_{3} \sum_{i=1}^{n} j_{i} k_{i}+\theta_{2} \sum_{i=1}^{n} j_{i} .
$$

The maximum likelihood estimates of $\theta_{1}, \theta_{2}$, and $\theta_{3}$ can be obtained by solving

$$
\begin{gathered}
\frac{\partial N(\theta) / \partial \theta_{1}}{N(\theta)}=\frac{1}{n} \sum_{i=1}^{n} j_{i}, \\
\frac{\partial N(\theta) / \partial \theta_{2}}{N(\theta)}=\frac{1}{n} \sum_{i=1}^{n} j_{i}, \\
\frac{\partial N(\theta) / \partial \theta_{3}}{N(\theta)}=-\frac{1}{n} \sum_{i=1}^{n} j_{i} k_{i},
\end{gathered}
$$

where $N(\theta)$ is given by (12).

The implicit nature of systems (36)-(38) suggests the numerical derivation of the MLE of parameters $\theta_{1}, \theta_{2}$, and $\theta_{3}$.

4.2. Pseudolikelihood Estimation for the Parameters. The pseudolikelihood method is an alternative estimation technique that does not include the normalizing constant (see Besag [34, 35] and Arnold and Strauss [36, 37]). The pseudolikelihood function can be written as

$$
\begin{aligned}
& \operatorname{PL}(\theta)=\prod_{i=1}^{n} P_{j \mid k}\left(j_{i} \mid k_{i}\right) P_{k \mid j}\left(k_{i} \mid j_{i}\right), \\
& \operatorname{PL}(\theta)=\prod_{i=1}^{n} \frac{\exp \left(-\theta_{2}+\theta_{3} k\right)-1}{\left(\exp \left(-\theta_{2}+\theta_{3} k\right)\right)^{j}} \frac{\exp \left(-\theta_{1}+\theta_{3} j\right)-1}{\left(\exp \left(-\theta_{1}+\theta_{3} j\right)\right)^{k}}
\end{aligned}
$$

Therefore, we have the following logarithmic form of the pseudolikelihood function:

$$
\begin{aligned}
\log \mathrm{PL}(\theta)= & \sum_{i=1}^{n} \log \left[\exp \left(-\theta_{2}+\theta_{3} k_{i}\right)-1\right] \\
& -\sum_{i=1}^{n} j_{i}\left(-\theta_{2}+\theta_{3} k_{i}\right) \\
& +\sum_{i=1}^{n} \log \left[\exp \left(-\theta_{1}+\theta_{3} j_{i}\right)-1\right] \\
& -\sum_{i=1}^{n} k_{i}\left(-\theta_{1}+\theta_{3} j_{i}\right) .
\end{aligned}
$$

The maximum pseudolikelihood estimates of $\theta_{1}, \theta_{2}$, and $\theta_{3}$ can be obtained by solving the following: 


$$
\begin{aligned}
& \frac{\partial \log \mathrm{PL}\left(\theta_{1}, \theta_{2}, \theta_{3}\right)}{\partial \theta_{1}}=-\sum_{i=1}^{n} \frac{\exp \left(-\theta_{1}+\theta_{3} j_{i}\right)}{\exp \left(-\theta_{1}+\theta_{3} j_{i}\right)-1}+\sum_{i=1}^{n} k_{i}, \\
& \frac{\partial \operatorname{logPL}\left(\theta_{1}, \theta_{2}, \theta_{3}\right)}{\partial \theta_{2}}=-\sum_{i=1}^{n} \frac{\exp \left(-\theta_{2}+\theta_{3} k_{i}\right)}{\exp \left(-\theta_{2}+\theta_{3} k_{i}\right)-1}+\sum_{i=1}^{n} x_{i}, \\
& \frac{\partial \operatorname{logPL}\left(\theta_{1}, \theta_{2}, \theta_{3}\right)}{\partial \theta_{3}}=\sum_{i=1}^{n} \frac{j_{i} \exp \left(-\theta_{1}+\theta_{3} j_{i}\right)}{\exp \left(-\theta_{1}+\theta_{3} j_{i}\right)-1}+\sum_{i=1}^{n} \frac{k_{i} \exp \left(-\theta_{2}+\theta_{3} k_{i}\right)}{\exp \left(-\theta_{2}+\theta_{3} k_{i}\right)-1}+2 \sum_{i=1}^{n} j_{i} k_{i} .
\end{aligned}
$$

\section{Application}

We consider a dataset in this paper which was obtained from Mitchell and Paulson [38] and is presented in Table 1. Utilizing these data, we should gauge and estimate the parameters $\theta_{1}, \theta_{2}$, and $\theta_{3}$ of class (4). The information includes flight aborts count data from 109 aircrafts, and the variables $J$ and $K$ represent the flight aborts in the first and second sequential six months of a one-year period.

The frequencies of the observed data provide several $(j$, $0)$ and $(0, k)$ data, indicating a negative correlation between $j$ and $k$. Therefore, we fit BPC, BP, BPL, and BNB distributions to the data since these distributions can be fitted to bivariate data with positive, zero, or negative correlation.

The statistic measures for the given data are $\bar{j}=0.62$, $s_{1}^{2}=1.03 ; \bar{k}=0.72, s_{2}^{2}=1.07, \operatorname{Cov}(j, k)=-0.169$, and $\rho=$ -0.16 . Table 2 presents the estimated parameters of the BPEC model and its mean square error (MSE).

The joint PMF of bivariate Poisson conditional distribution can be defined as $\operatorname{BPC}\left(\lambda_{1}, \lambda_{2}, \lambda_{3}\right)$ and defined as follows (Arnold and Strauss [36]):

$$
\begin{aligned}
P(J=j, K=k) & =C\left(\lambda_{1}, \lambda_{2}, \lambda_{3}\right) \frac{\lambda_{1}^{j} \lambda_{2}^{k} \lambda_{3}^{j k}}{j ! k !} \\
j, k & =0,1,2, \ldots, \lambda_{1}, \lambda_{2}>0,0<\lambda_{3} \leq 1,
\end{aligned}
$$

where $C\left(\lambda_{1}, \lambda_{2}, \lambda_{3}\right)$ is constant. The conditionals $K \mid J$ and $J \mid K$ are $\lambda_{2} \lambda_{3}^{j}$ and $\lambda_{1} \lambda_{3}^{k}$, respectively.

The joint PMF of BP $\left(\lambda_{1}, \lambda_{2}, \alpha\right)$ distribution is (Lakshminarayana et al. [39])

$$
\begin{aligned}
& P(J=j, K=k)=e^{-\lambda_{1}-\lambda_{2}} \frac{\lambda_{1}^{j} \lambda_{2}^{k}}{j k}\left[1+\alpha\left(e^{-j}-e^{-c \lambda_{1}}\right)\left(e^{-k}-e^{-c \lambda_{2}}\right)\right], \\
& j, k=0,1,2, \ldots, \lambda_{1}, \lambda_{2}>0,
\end{aligned}
$$

where $c=1-e^{-1}, \quad E(J)=\operatorname{Var}(J)=\lambda_{1}, E(K)=\operatorname{Var}(K)=$ $\lambda_{2}, \operatorname{Cov}(J, K)=\alpha \lambda_{1} \lambda_{2} c^{2} e^{-c\left(\lambda_{1}+\lambda_{2}\right)}$, and $\alpha$ can be chosen such that $P\left(Y_{1}, Y_{2}\right)$ will be the PMF.

The joint PMF of $\operatorname{BPL}\left(\theta_{1}, \theta_{2}, m_{1}, m_{2}, \gamma\right)$ distribution is (Zamani et al. [40])

$$
\begin{array}{r}
P(J=j, K=k)=\frac{\theta_{1}^{2}\left(j+\theta_{1}+2\right) \theta_{1}^{2}\left(j+\theta_{1}+2\right)}{\left(\theta_{1}+1\right)^{j+3}\left(\theta_{2}+1\right)^{k+3}} \\
\cdot\left[1+\alpha\left(e^{-j}-c_{1}\right)\left(e^{-k}-c_{2}\right)\right], \\
j, k=0,1,2, \ldots, \theta_{1}, \theta_{2}>0,
\end{array}
$$

where

$$
\begin{aligned}
& c_{1}=E\left(e^{-J}\right)=\frac{\theta_{1}^{2}}{1+\theta_{1}} \frac{\left(\theta_{1}+2-e^{-1}\right)}{\left(\theta_{1}-e^{-1}+1\right)^{2}}, \\
& c_{2}=E\left(e^{-K}\right)=\frac{\theta_{2}^{2}}{1+\theta_{2}} \frac{\left(\theta_{2}+2-e^{-1}\right)}{\left(\theta_{2}-e^{-1}+1\right)^{2}} .
\end{aligned}
$$

The joint $\operatorname{PMF}$ of $\operatorname{BNB}\left(\theta_{1}, \theta_{2}, m_{1}, m_{2}, \gamma\right)$ distribution is (Famoye [41])

$$
\begin{aligned}
P(J & =j, K=k) \\
& =\left(\begin{array}{c}
m_{1}^{-1}+j-1 \\
k
\end{array}\right) \theta_{1}^{j}\left(1-\theta_{1}\right)^{m_{1}^{-1}}\left(\begin{array}{c}
m_{2}^{-1}+j-1 \\
k
\end{array}\right) \theta_{2}^{k}\left(1-\theta_{2}\right)^{m_{2}^{-1}}\left[1+\gamma\left(e^{-j}-c_{1}\right)\left(e^{-k}-c_{2}\right)\right], \\
j, k & =0,1,2, \ldots, \theta_{1}, \theta_{2}>0,0<m_{1}, m_{2}<1,
\end{aligned}
$$


TABLE 1: The flight abort check information starting with 109 aircraft.

\begin{tabular}{ccccccc}
\hline$j$ & & \multicolumn{5}{c}{$K$} \\
& 0 & 1 & 2 & 3 & 4 & $\sum$ \\
\hline 0 & 34 & 20 & 4 & 6 & 4 & 68 \\
1 & 17 & 7 & 0 & 0 & 0 & 24 \\
2 & 6 & 4 & 1 & 0 & 0 & 11 \\
3 & 0 & 4 & 0 & 0 & 0 & 4 \\
4 & 0 & 0 & 0 & 0 & 0 & 0 \\
5 & 2 & 0 & 0 & 0 & 0 & 2 \\
$\sum$ & 59 & 35 & 5 & 6 & 4 & 109 \\
\hline
\end{tabular}

TABLE 2: Estimation of parameters of the BPEC.

\begin{tabular}{lcccc}
\hline Parameters & MLE & MSE & MPLE & MSE \\
\hline$\theta_{1}$ & -0.6712 & 0.271675 & -0.932498 & 0.000506142 \\
$\theta_{2}$ & -0.7664 & 0.0709704 & -0.824366 & 0.000593723 \\
$\theta_{3}$ & -1.6437 & 0.0206583 & 0.57096 & 0.00167806 \\
\hline
\end{tabular}

where

$$
\begin{aligned}
& c_{1}=E\left(e^{-J}\right)=\left(\frac{1-\theta_{1}}{1-\theta_{1} e^{-1}}\right)^{m_{1}^{-1}}, \\
& c_{2}=E\left(e^{-K}\right)=\left(\frac{1-\theta_{2}}{1-\theta_{2} e^{-1}}\right)^{m_{2}^{-1}},
\end{aligned}
$$

and the mean, variance, and covariance are

$$
\begin{aligned}
E(J) & =m_{1}^{-1} \frac{\theta_{1}}{1-\theta_{1}}, \\
E(K) & =m_{2}^{-1} \frac{\theta_{2}}{1-\theta_{2}}, \\
\operatorname{Var}(J) & =m_{1}^{-1} \frac{\theta_{1}}{\left(1-\theta_{1}\right)^{2}}, \\
\operatorname{Var}(K) & =m_{2}^{-1} \frac{\theta_{2}}{\left(1-\theta_{2}\right)^{2}}, \\
\operatorname{Cov}(J, K) & =\gamma c_{1} c_{2} \prod_{i}\left(\frac{m \alpha_{i}^{-1} \theta_{i} e^{-1}}{1-\theta_{i} e^{-1}}-\frac{m_{i}^{-1} \theta_{i}}{1-\theta_{i}}\right) .
\end{aligned}
$$

We used the Mathematica package to estimate the parameters of BPEC distribution.

The new distribution BPEC is more appropriate as we can see in Table 3 as compared to the BPC, BP, BPL, and $\mathrm{BNB}$ distributions, where the BPEC distribution gives the largest value for the AIC and BIC statistics compared to other models.
TABLE 3: Parameter estimators and AIC and BIC of BPEC, BPC, BP, $\mathrm{BNB}$, and BPL distributions.

\begin{tabular}{lccccc}
\hline Model & Parameter & MLE & $\begin{array}{c}\text { Log- } \\
\text { likelihood }\end{array}$ & AIC & BIC \\
\hline \multirow{3}{*}{ BPEC } & $\theta_{1}$ & -0.6712 & & & \\
& $\theta_{2}$ & -0.7664 & -114.821 & -120.821 & -121.858 \\
& $\theta_{3}$ & -1.6437 & & & \\
BPC & $\lambda_{1}$ & 0.14388 & & & \\
& $\lambda_{2}$ & 0.208112 & -252.078 & -258.078 & -259.115 \\
& $\lambda_{3}$ & 0.426338 & & & \\
BP & $\lambda_{1}$ & 0.6129 & & & \\
& $\lambda_{2}$ & 0.7131 & -254.99 & -260.99 & -262.027 \\
& $\alpha$ & -0.9290 & & & \\
BPL & $\theta_{1}$ & 2.1165 & & & \\
& $\theta_{2}$ & 1.8607 & -244.62 & -250.62 & -251.657 \\
& $\alpha$ & -1.0363 & & & \\
BNB & $\theta_{1}$ & 0.4045 & & & \\
& $\theta_{2}$ & 0.3138 & & & \\
& $m_{1}$ & 1.0977 & -244.27 & -254.27 & -255.998 \\
& $m_{2}$ & 0.6305 & & & \\
\hline
\end{tabular}

\section{Conclusion}

In this work, a BPEC model is presented by determining conditional discrete Poisson exponential distributions. Therefore, we obtained the statistical properties and special classes for BPEC distribution. The estimation of BPEC parameters through the techniques for MLE and MPLE is presented. In view of the findings presented in Table 1, the MPLE is better than MLE because the MPLE technique uses conditional distributions which in our case do not suffer from the problem caused by the normalizing constant. Moreover, the AIC and BIC depict that BPEC distribution adequately fits the considered dataset compared to the BPC, $\mathrm{BP}, \mathrm{BPL}$, and BNB distributions.

\section{Data Availability}

The data used to support the findings of this study are included within the article.

\section{Conflicts of Interest}

The authors declare that they have no conflicts of interest.

\section{Acknowledgments}

This study was supported by Taif University Researchers Supporting Project (TURSP-2020/279), Taif University, Taif, Saudi Arabia.

\section{References}

[1] S.-H. Park, "Regression and correlation in a bivariate distribution with different marginal densities," Technometrics, vol. 12, no. 3, pp. 687-691, 1970.

[2] X. Wu and K. C. Yuen, "A discrete-time risk model with interaction between classes of business," Insurance: Mathematics and Economics, vol. 33, no. 1, pp. 117-133, 2003. 
[3] K. C. Yuen, J. Guo, and X. Wu, "On the first time of ruin in the bivariate compound Poisson model," Insurance: Mathematics and Economics, vol. 38, no. 2, pp. 298-308, 2006.

[4] L. B. Morata, "A priori ratemaking using bivariate Poisson regression models," Insurance: Mathematics and Economics, vol. 44, no. 1, pp. 135-141, 2009.

[5] T. Cacoullos and H. Papageorgiou, "On some bivariate probability models applicable to traffic accidents and fatalities," International Statistical Review/Revue Internationale de Statistique, vol. 48, no. 3, pp. 345-356, 1980.

[6] H. Papageorgiou, "On a bivariate Poisson-geometric distribution," Applicationes Mathematicae, vol. 18, no. 4, pp. 541-547, 1985.

[7] H. Papageorgiou, "Some remarks on the D compound Poisson distribution," Statistical Papers, vol. 36, no. 1, pp. 371-375, 1995.

[8] T. D. Miethe, T. C. Hart, and W. C. Regoeczi, "The conjunctive analysis of case configurations: an exploratory method for discrete multivariate analyses of crime data," Journal of Quantitative Criminology, vol. 24, no. 2, pp. 227-241, 2008.

[9] A. J. Lee, "Modeling scores in the premier league: is Manchester United really the best?" Chance, vol. 10, no. 1, pp. 15-19, 1997.

[10] D. Karlis and I. Ntzoufras, "On modelling soccer data," The Student, vol. 3, pp. 229-244, 2000.

[11] I. McHale and P. Scarf, "Modelling the dependence of goals scored by opposing teams in international soccer matches," Statistical Modelling, vol. 11, no. 3, pp. 219-236, 2011.

[12] T. M. Ahooyi, J. E. Arbogast, U. G. Oktem, W. D. Seider, and M. Soroush, "Estimation of complete discrete multivariate probability distributions from scarce data with application to risk assessment and fault detection," Industrial \& Engineering Chemistry Research, vol. 53, no. 18, pp. 7538-7547, 2014.

[13] A. W. Marshall and I. Olkin, "Bivariate distributions generated from Pólya-Eggenberger urn models," Journal of Multivariate Analysis, vol. 35, no. 1, pp. 48-65, 1990.

[14] A. Mishra, "A generalised bivariate binomial distribution applicable in four-fold sampling," Communications in Statistics-Theory and Methods, vol. 25, no. 8, pp. 1943-1956, 1996.

[15] G. Özel, "A bivariate compound Poisson model for the occurrence of foreshock and aftershock sequences in Turkey," Environmetrics, vol. 22, no. 7, pp. 847-856, 2011.

[16] G. Ozel, "On certain properties of a class of bivariate compound Poisson distributions and an application on earthquake data," Revista Colombiana de Estadística, vol. 34, pp. 545-566, 2011.

[17] G. Ozel, "On the moment characteristics of univariate compound Poisson and bivariate compound Poisson processes with applications," Revista Colombiana de Estadística, vol. 36, pp. 59-77, 2013.

[18] C. H. Reilly and N. Sapkota, "A family of composite discrete bivariate distributions with uniform marginals for simulating realistic and challenging optimization-problem instances," European Journal of Operational Research, vol. 241, no. 3, pp. 642-652, 2015.

[19] H. Lee and J. H. Cha, "On two general classes of discrete bivariate distributions," The American Statistician, vol. 69, no. 3, pp. 221-230, 2015.

[20] X. Jiang, J. Chu, and S. Nadarajah, "New classes of discrete bivariate distributions with application to football data," Communications in Statistics-Theory and Methods, vol. 46, no. 16 , pp. 8069-8085, 2016.

[21] E. Castillo and J. Galambos, "Bivariate distributions with normal conditionals," in Proceedings of the IASTED
International Symposium, Cairo, M. H. Hamza, Ed., pp. 5962, Acta Press, Anaheim, CA, USA, 1987.

[22] E. Castillo and J. Galambos, Bivariate Distributions with Weibull Conditionals, Technical Report, Department of Mathematics, Temple University, Philadelphia, PA, USA, 1987.

[23] E. Castillo and J. Galambos, "Conditional distributions and the bivariate normal distribution," Metrika, vol. 36, no. 1, pp. 209-214, 1989.

[24] B. C. Arnold, "Bivariate distributions with Pareto conditionals," Statistics \& Probability Letters, vol. 5, no. 4, pp. 263-266, 1987.

[25] B. C. Arnold, E. Castillo, and J. M. Sarabia, "Multivariate distributions with generalized Pareto conditionals," Statistics \& Probability Letters, vol. 17, no. 5, pp. 361-368, 1993.

[26] B. C. Arnold, E. Castillo, and J. M. Sarabia, "Conditional specifiecation of statistical models," Springer Series in Statistics, Springer Verlag, New York, NY, USA, 1999.

[27] B. C. Arnold, E. Castillo, and J. M. Sarabia, "Conditionally specified distributions: an introduction (with discussion)," Statistical Science, vol. 16, no. 3, pp. 249-274, 2001.

[28] A. Kottas, K. Adamidis, and S. Loukas, "Bivariate distributions with Pearson type VII conditionals," Annals of the Institute of Statistical Mathematics, vol. 51, no. 2, pp. 331-344, 1999.

[29] M. Gharib and B. I. Mohammed, "A new class of bivariate distributions with exponential and gamma conditionals," International Journal of Reliability and Applications, vol. 15, no. 2, pp. 111-123, 2014.

[30] J. M. Sarabia, E. Gómez-Déniz, and F. J. Vázquez-Polo, “On the use of conditional specification models in claim count distributions: an application to bonus-malus systems," ASTIN Bulletin, vol. 34, no. 1, pp. 85-98, 2004.

[31] J. M. Sarabia, E. Castillo, E. Gomez-Deniz, and F. J. VazquezPolo, "A class of conjugate priors for log-normal claims based on conditional specification," Journal of Risk \& Insurance, vol. 72, no. 3, pp. 479-495, 2005.

[32] A. Fazal and S. Bashir, "Family of Poisson distribution and its application," International Journal of Applied Mathematics \& Statistical Sciences, vol. 6, no. 4, pp. 1-18, 2017.

[33] J. Aczel, Lectures on Functional Equations and Their Applications, Academic Press, New York, NY, USA, 1966.

[34] J. Besag, "Statistical analysis of non-lattice data," The Statistician, vol. 24, no. 3, pp. 179-195, 1975.

[35] J. Besag, "Efficiency of pseudolikelihood estimation for simple Gaussian fields," Biometrika, vol. 64, no. 3, pp. 616-618, 1977.

[36] B. C. Arnold and D. Strauss, "Bivariate distributions with exponential conditionals," Journal of the American Statistical Association, vol. 83, no. 402, pp. 522-527, 1988.

[37] B. Arnold and D. Strauss, "Pseudolikelihood estimation," Sankhya Series B, vol. 53, pp. 233-243, 1988.

[38] C. R. Mitchell and A. S. Paulson, "A new bivariate negative binomial distribution," Naval Research Logistics Quarterly, vol. 28, no. 3, pp. 359-374, 1981.

[39] J. Lakshminarayana, S. N. N. Pandit, and K. Srinivasa Rao, "On a bivariate Poisson distribution," Communications in Statistics-Theory and Methods, vol. 28, no. 2, pp. 267-276, 1999.

[40] H. Zamani, P. Faroughi, and N. Ismail, "Bivariate PoissonLindley distribution with application," Journal of Mathematics and Statistics, vol. 11, no. 1, pp. 1-6, 2015.

[41] F. Famoye, "On the bivariate negative binomial regression model," Journal of Applied Statistics, vol. 37, no. 6, pp. 969-981, 2010. 